TPI-MINN-96-28/T

NUC-MINN-96-22/T

HEP-MINN-96-1524

\title{
The BFKL Equation from the Wilson Renormalization Group
}

\author{
Jamal Jalilian-Marian ${ }^{1}$, Alex Kovner ${ }^{1}$, Andrei Leonidov² ${ }^{2}$ and Heribert \\ Weigert ${ }^{3}$ \\ ${ }^{1}$ Physics Department, University of Minnesota \\ 116 Church st. S.E., Minneapolis, MN 55455, USA \\ ${ }^{2}$ Theoretical Physics Department, P.N. Lebedev Physics Institute, \\ 117924 Leninsky pr. 53 Moscow, Russia \\ ${ }^{3}$ University of Cambridge, Cavendish Laoratory, HEP, Madingley Road, Cambridge \\ CB3 OHE UK
}

\begin{abstract}
We discuss the Wilson renormalization group approach to the effective action for low $x$ physics. It is shown that in the linearized, weak field regime the RG equation reduces to the BFKL equation for the evolution of the unintegrated gluon density. We discuss the relation of this approach with that of Lipatov.
\end{abstract}




\section{Introduction}

Physics of dense gluon systems is one of the most rapidly growing branches of quantum theory of strong interactions (see [1] for a recent review). The recent wave of interest was sparked by experimental data on deep inelastic scattering [2] showing a considerable growth of gluon density in a proton at small Bjorken $x$.

Theoretical understanding of small $x$ physics is a fascinating problem in itself because it envolves a delicate blend of perturbative and nonperturbative QCD physics. On the one hand, due to large partonic densities, the QCD coupling constant in this region is expected to be small. On the other hand however, the large density must also lead to appearance of some collective effects (perhaps similar to the ones often disussed in the framework of the high temperature QCD) which should be taken into account nonperturbatively.

The perturbative paradigm of the low $x$ gluon physics is the famous BFKL equation [3]. It resums all contributions of the form $\left(\alpha_{s} \ln 1 / x\right)^{n}$ in the naive perturbative series. This is a linear equation, which describes the evolution with $x$ of the unintegrated gluon density $\varphi(y=\ln 1 / x, \mathbf{k})$, related to the standard gluon distribution function $x g\left(x, Q^{2}\right)$ by

$$
x g\left(x, Q^{2}\right)=\int_{0}^{Q^{2}} \frac{d^{2} k}{\mathbf{k}^{2}} \varphi(x, \mathbf{k})
$$

The equation is

$$
\frac{\partial \varphi\left(y, \mathbf{q}^{2}\right)}{\partial y}=\frac{\alpha_{s} N_{c}}{\pi^{2}} \int d^{2} k K(\mathbf{q}, \mathbf{k}) \varphi(y, \mathbf{k})
$$

The action of the BFKL kernel on a function reads

$$
\int d^{2} k K(\mathbf{q}, \mathbf{k}) \varphi(y, \mathbf{k})=\int d^{2} k\left[K_{r e}(\mathbf{q}, \mathbf{k})+K_{v i r t}(\mathbf{q}, \mathbf{k})\right] \varphi(y, \mathbf{k})
$$

where

$$
\int d^{2} k K_{r e}(\mathbf{q}, \mathbf{k}) \varphi(y, \mathbf{k})=\mathbf{q}^{2} \int d^{2} k \frac{1}{\mathbf{k}^{2}(\mathbf{q}-\mathbf{k})^{2}} \varphi(y, \mathbf{k})
$$


is the contribution of real gluon emission to the evolution in $\alpha_{s} \ln 1 / x$ and

$$
\int d^{2} k K_{v i r t}(\mathbf{q}, \mathbf{k}) \varphi(y, \mathbf{k})=-\frac{\mathbf{q}^{2}}{2} \int d^{2} k \frac{1}{\mathbf{k}^{2}(\mathbf{q}-\mathbf{k})^{2}} \varphi(y, \mathbf{q})
$$

is the correspoding virtual contribution which eventually leads (after resummation) to the gluon reggeization [4], [5].

The solution of the BFKL equation exhibits a powerlike growth at small $x$,

$$
\varphi(x, \mathbf{k}) \sim\left(\frac{1}{x}\right)^{4 \bar{\alpha}_{s} \ln 2}
$$

which leads to apparent violation of unitarity at very small $x$. It was argued a long time ago [6] that eventually the system should enter a new regime, where the rate of growth of the gluon density slows down and eventually saturates, thus curing a potential conflict with unitarity of the underlying scattering. The responsibility for the slow down and the saturation should lie with the nonperturbative finite density effects which are left out entirely from the BFKL evolution. The restoration of unitarity in high energy (density) limit of QCD remains an outstanding problem which remains unsolved although several approaches are being explored in the literature [4], [7], [8].

This paper is based on the approach first proposed by McLerran and Venugopalan [9] in the context of ultrarelativistic heavy ion collisions. The idea in [9] is that there is a regime of high density and weak coupling in which semiclassical methods should apply. It is therefore suggested that the leading small $x$ glue structure of the nucleus is due to the classical gluon field which is created by the random color charges of energetic on-shell partons. The nonlinearities of the Yang - Mills equations exhibit themselves already on this classical level and it is therefore possible that they provide the necessary saturation mechanism at low $x$. It is to be stressed that this approach is intrinsically nonperturbative since both the color charge density and the classical glue fields (gluon density) are not considered to be small when solving the classical equations. 
Originally only the valence quarks were considered as the sources of the color field [9]. It turned out that the quantum corrections to this approximation [10 are big at low $x$. Physically this is understandable since at asymptotically small $x$ the source for the glue field should include not only the valence quarks but all "on shell" partons with longitudinal momenta $p^{+}>x P^{+}$(where $P^{+}$is the momentum of a hadron).

Experimentallly it is known [2] that the dominant contribution to the proton structure function at small Bjorken $x$ is given by gluons. Their relative contribution, compared to the valence quark contribution, grows when $x$ becomes smaller.

At small $x$ therefore gluons give the main contribution to the color charge density. The typical corrections to the leading order semiclassical results were of order of $\alpha_{s} \ln 1 / x$ showing the necessity of resumming the leading $\ln 1 / x$ contribution.

The Wilson Renormalization Group approach to this resummation was developed in [11]. The idea is to generate an effective Lagrangian for low $x$ fields by integrating out all quantum fluctuations around the classical background with longitudinal momenta $p^{+}>$ $x P^{+}$. The low $x$ glue field then should be determined by solving classical equations that follow from this effective Lagrangian. The main effect of this integrating out procedure is the change in the color charge density distribution in the effective Lagrangian. It was also realized in 11] that when formulated in this way, the effective Lagrangian approach should be applicable not only to heavy ions but to ordinary hadrons as well. The double logarithmic asymtotics in $\ln q^{2} \ln 1 / x$ was considered within this framework as the first step in [11] . The validity of this RG approach should not depend on whether the classical background glue field is strong or not. It is most interesting therefore to apply it to the strong field - high density situation. However, before plunging into the nonlinear realm of strong fields, it is natural to ask whether the approach of [11] at a simple linear level reproduces the BFKL evolution equation. This is in fact a crucial test for the whole semiclassical philosophy, the importance of which was recognized already in [9]. The 
answer to this question turns out to be affirmative.

Derivation of BFKL evolution equation from the renormalization group improved low $x$ effective action is the main subject of this paper. We shall also comment on the relation of this approach to that of Lipatov 4 . The paper is organized as follows. In Section 2 we describe in some detail the structure of the low $x$ effective action and the renormalization group procedure. In Section 3 we calculate the RG equation in the weak field limit and show that in this limit it reduces to the BFKL equation for the DIS gluon distribution function. In Section 4 we comment on the relation of our approach to that of Lipatov and make some closing remarks.

\section{The effective action and the renormalization group}

The starting point of our approach is the following action given in the Light Cone gauge $A^{+}=0$

$$
\begin{aligned}
S & =i \int d^{2} x_{t} F\left[\rho^{a}\left(x_{t}\right)\right] \\
& -\int d^{4} x \frac{1}{4} G^{2}+\frac{i}{N_{c}} \int d^{2} x_{t} d x^{-} \delta\left(x^{-}\right) \rho^{a}\left(x_{t}\right) \operatorname{tr} T_{a} W_{-\infty, \infty}\left[A^{-}\right]\left(x^{-}, x_{t}\right)
\end{aligned}
$$

Here $G^{\mu \nu}$ is the gluon field strength tensor

$$
G_{a}^{\mu \nu}=\partial^{\mu} A_{a}^{\nu}-\partial^{\nu} A_{a}^{\mu}+g f_{a b c} A_{b}^{\mu} A_{c}^{\nu}
$$

$T_{a}$ are the $S U(N)$ color matrices in the adjoint representation, and $W$ is the path ordered exponential along the $x^{+}$direction in the adjoint representation of the $S U\left(N_{c}\right)$ group

$$
W_{-\infty, \infty}\left[A^{-}\right]\left(x^{-}, x_{t}\right)=P \exp \left[-i g \int d x^{+} A_{a}^{-}\left(x^{-}, x_{t}\right) T_{a}\right]
$$

Even though the functional $S[\rho, A]$ has an imaginary part, we will use the term action when referring to it since the average of any gluonic operator $O(A)$ in the hadron is 
calculated as

$$
<O>=\frac{\int\left[D \rho^{a}\right]\left[D A_{a}^{\mu}\right] O(A) \exp \{i S[\rho, A]\}}{\int\left[D \rho^{a}\right]\left[D A_{a}^{\mu}\right] \exp \{i S[\rho, A]\}}
$$

The exponential of the imaginary part of the action

$$
\operatorname{Im} S=\int d^{2} x_{t} F\left[\rho^{a}\right]
$$

can be thought of as a kind of "free energy". The "Bolzmann factor"

$$
\exp \left\{-\int d^{2} x_{t} F\left[\rho^{a}\right]\right\}
$$

appearing in (10) controls the statistical weight of a particular configuration of the two dimensional color charge density $\rho^{a}\left(x_{t}\right)$ inside the hadron.

Since the action (7) is a little different than the one used in [9], [11] some clarifying remarks are in order here. In the McLerran - Venugopalan (MV) action [9], 11] the "free energy" was taken to be a gaussian of the form

$$
F\left[\rho\left(x_{t}\right)\right]=\frac{1}{2 \mu^{2}} \rho_{a}^{2}\left(x_{t}\right)
$$

The dimensional constant $\mu^{2}$ then has the meaning of the average color charge density squared per unit area. This form of the "free energy" is valid as long as the charge density is large and the color charges that build it up are randomly distributed in color space.

In this paper we prefer not to specify $F[\rho]$ explicitly, but rather think of it as a general positive definite functional. This we do for two reasons. First, for the purpose of derivation of the BFKL equation we will not need the explicit form of $F$. Second, the low $x$ renormalization group procedure that we employ, in the general nonlinear case results in an equation of the form

$$
\frac{d}{d y} F[\rho]=\alpha \Delta[\rho]
$$


so that the functional form of $F$ changes as one considers lower and lower values of $x$. In fact our expectation is that (14) will have a fixed point as $x \rightarrow 0$, thereby providing a mechanism for unitarization.

Another element which is different in the action (7) from the MV action is the last term which involves the Wilson line along the $x^{+}$direction. The origin of this term is easy to understand. It is a natural gauge invariant extension of the abelian coupling of the glue field to the external color charge density $\rho^{a}$. The equation of motion that follows from this action is

$$
D_{\mu} G^{\mu \nu}=J^{+} \delta^{\nu+}
$$

with

$$
J_{a}^{+}(x)=\frac{g}{N_{c}} \delta\left(x^{-}\right) \rho^{b}\left(x_{t}\right) \operatorname{tr}\left[T_{b} W_{-\infty, x^{+}}\left[A^{-}\right] T_{a} W_{x^{+}, \infty}\left[A^{-}\right]\right]
$$

Expanding this expression for the current to lowest order in the field $A^{-}$yields

$$
J_{a}^{+}(x)=g \delta\left(x^{-}\right) \rho^{a}\left(x_{t}\right)
$$

which is the form of the current used in [9], [10]. As explained in [9], this form is only valid in the gauge $A^{-}\left(x^{-}=0\right)=0$. In more general gauges the current should satisfy the covariant conservation condition

$$
D^{-} J^{+}=0
$$

It is straightforward to check that our current (16) is indeed covariantly conserved. This is a direct consequence of the fact that the action (7) in the light cone gauge $A^{+}=0$ is still gauge invariant under the residual gauge transformations with gauge functions which do not depend on $x^{-}$and vanish at $x^{+} \rightarrow \pm \infty$. Under an infinitesimal transformation with such a gauge function $\lambda^{a}\left(x_{t}, x^{+}\right)$we have from the Wilson line in the action

$$
0=\delta S_{W}=\int \lambda^{a}\left(D^{-} \frac{\delta S}{\delta A_{a}^{-}}\right) \equiv \int \lambda^{a}\left(D^{-} J^{+}\right)^{a}
$$


Thus the gauge invariance is equivalent to covariant conservation of the current?.

Now, the MV approximation for calculating the path integral in eq.(10) is to find the classical solution for the equations of motion eq.(155) at fixed $\rho$, and then to average over the charge density distribution with the "Bolzmann weight" eq.(12). The classical solution for any fixed $\rho\left(x_{t}\right)$ has the structure

$$
\begin{aligned}
A_{c l}^{-} & =0 \\
A_{c l}^{i} \equiv b^{i} & =\theta\left(x^{-}\right) \alpha^{i}\left(x_{t}\right)
\end{aligned}
$$

The presence of the extra terms in our action as compared to ref. [9] does not change the classical solution since the extra terms involve only $A^{-}$which vanishes on the classical solution anyway. The quantum corrections to this classical approximation are large at small longitudinal momenta. To resum these large corrections we follow the renormalization group procedure described in [11].

Let us introduce the following decomposition of the gauge field:

$$
A_{\mu}^{a}(x)=b_{\mu}^{a}(x)+\delta A_{\mu}^{a}(x)+a_{\mu}^{a}(x)
$$

where $b_{\mu}^{a}(x)$ is the solution of the classical equations of motion, $\delta A_{\mu}^{a}(x)$ is the fluctuation field containing longitudinal momentum modes $q^{+}$such that $P_{n}^{+}<q^{+}<P_{n-1}^{+}$while $a$ is a soft field with momenta $k^{+}<P_{n}^{+}$, with respect to which the effective action is computed. The initial path integral is formulated with the longitudinal momentum cutoff on the field $\delta A, q^{+}<P_{n-1}^{+}$. The effective action for $a^{\mu}$ is calculated by integrating over the fluctuations $\delta A$. This integration is performed within the assumption that

\footnotetext{
${ }^{1}$ The charge density $\rho\left(x_{t}\right)$ has the meaning of a transition matrix element. Roughly speaking,

$$
\rho^{a}\left(x_{t}\right) \sim<\Psi_{i}\left|\int d x^{-} j^{a}\left(x_{t}, x^{-}\right)\right| \Psi_{f}>
$$

where $j^{a}\left(x_{t}, x^{-}\right)$is the charge density operator of the energetic partons, and $\mid \Psi_{i}>$ and $\left|\Psi_{f}\right\rangle$ are their wave functions at $x^{+} \rightarrow-\infty$ and $x^{+} \rightarrow \infty$ respectively. It is therefore natural that $\rho\left(x_{t}\right)$ is gauge invariant under gauge transformations that vanish at infinity. Under $x^{+}$independent gauge transformations it transforms as an adjoint field.
} 
the fluctuations are small as compared to the classical fields $b_{\mu}^{a}$. More quantitatively, at each step of this RG procedure the scale $P_{n}^{+}$is chosen such that $\ln \frac{P_{n-1}^{+}}{P_{n}^{+}}>1$, but $\alpha_{s} \ln \frac{P_{n-1}^{+}}{P_{n}^{+}}<<1$. Expanding the action around the classical solution $b_{\mu}^{a}(x)$ and keeping terms of the first and second order in $\delta A$ we get

$$
S=-\frac{1}{4} G(a)^{2}-\frac{1}{2} \delta A_{\mu}\left[\mathrm{D}^{-1}(\rho)\right]^{\mu \nu} \delta A_{\nu}+g a^{-} \rho^{\prime}+O\left(\left(a^{-}\right)^{2}\right)+i F[\rho]
$$

where

$$
\rho^{\prime}=\rho+\delta \rho_{1}+\delta \rho_{2}
$$

with

$$
\begin{aligned}
\delta \rho_{1}^{a}\left(x_{t}, x^{+}\right) & =-2 f^{a b c} \alpha_{i}^{b} \delta A_{i}^{c}\left(x^{-}=0\right) \\
& -\frac{g}{2} f^{a b c} \rho^{b}\left(x_{t}\right) \int d y^{+}\left[\theta\left(y^{+}-x^{+}\right)-\theta\left(x^{+}-y^{+}\right)\right] \delta A^{-c}\left(y^{+}, x_{t}, x^{-}=0\right)
\end{aligned}
$$

and

$$
\begin{aligned}
\delta \rho_{2}^{a}(x) & =-f^{a b c}\left[\partial^{+} \delta A_{i}^{b}(x)\right] \delta A_{i}^{c}(x) \\
& -\frac{g^{2}}{N_{c}} \rho^{b}\left(x_{t}\right) \int d y^{+} \delta A^{-c}\left(y^{+}, x_{t}, x^{-}=0\right) \int d z^{+} \delta A^{-d}\left(z^{+}, x_{t}, x^{-}=0\right) \\
& \times\left[\theta\left(z^{+}-y^{+}\right) \theta\left(y^{+}-x^{+}\right) \operatorname{tr} T^{a} T^{c} T^{d} T^{b}\right. \\
& +\theta\left(x^{+}-z^{+}\right) \theta\left(z^{+}-y^{+}\right) \operatorname{tr} T^{a} T^{b} T^{c} T^{d} \\
& \left.+\theta\left(z^{+}-x^{+}\right) \theta\left(x^{+}-y^{+}\right) \operatorname{tr} T^{a} T^{d} T^{b} T^{c}\right]
\end{aligned}
$$

The first term in both $\delta \rho_{1}^{a}$ and $\delta \rho_{2}^{a}$ is coming from expansion of $G^{2}$ in the action while the rest of the terms proportional to $\rho$ are from the expansion of the Wilson line term. The three terms correspond to different time ordering of the fields. Since the longitudinal momentum of $a^{-}$is much lower than of $\delta A$, we have only kept the eikonal coupling (the coupling to $a^{-}$only), which gives the leading contribution in this kinematics. The inverse propagator $\left[\mathrm{D}^{-1}\right]^{\mu \nu}$ is given by

$$
\left[\mathrm{D}^{-1}\right]_{a b}^{i j}(x, y)=\left[D^{2}(b) \delta^{i j}+D^{i}(b) D^{j}(b)\right]_{a b} \delta^{(4)}(x, y)
$$




$$
\begin{aligned}
{\left[\mathrm{D}^{-1}\right]_{a b}^{i+}(x, y) } & =-\left[\partial_{x}^{+} D_{a b}^{i}(b) \delta^{(4)}(x, y)+2 f_{a b c} \alpha_{c}^{i}\left(x_{t}\right) \delta\left(x^{-}\right) \delta\left(y^{-}\right) \delta^{(2)}\left(x_{t}, y_{t}\right) \delta\left(x^{+}, y^{+}\right)\right] \\
{\left[\mathrm{D}^{-1}\right]_{a b}^{++}(x, y) } & =\left(\partial^{+}\right)^{2} \delta_{a b} \delta^{(4)}(x, y)+f_{a b c} \rho^{c}\left(x_{t}\right) \delta\left(x^{-}\right) \delta\left(y^{-}\right) \theta\left(x^{+}-y^{+}\right) \delta^{(2)}\left(x_{t}, y_{t}\right)
\end{aligned}
$$

Two things should be noted here. First, formally $\delta \rho$ is a function of $x^{+}$and $x^{-}$as well as $x_{t}$. However, it is a function of $\delta A$ 's which only have longitudinal momenta much larger than the momenta in the soft field $a$. The (light cone) time variation scale of $\delta \rho$ is therefore $\frac{1}{q^{-}} \sim \frac{q^{+}}{q_{t}^{2}}$ and is much larger than the typical time variation scale of the on shell modes of the field $a$. From this point of view $\delta \rho$ is therefore for all practical purposes (light cone) time independent. As for $x^{-}$dependence, the only term which does not have an explicit $\delta\left(x^{-}\right)$is the first term in eq.(25). However remember that we are only interested in its low longitudinal momentum components since it couples directly to $a^{-}$in the effective action. So, in momentum space we are interested in $f^{a b c} \int d q^{+}\left[q^{+} \delta A_{i}^{b}\left(q^{+}\right)\right] \delta A_{i}^{c}\left(-q^{+}+k^{+}\right)$. Since the leading logarithmic contributions comes from the region $q^{+}>>k^{+}$, to this accuracy this expression does not depend on $k^{+}$and is therefore also $\delta\left(x^{-}\right)$in coordinate space. Therefore $\delta \rho$ behaves as an honest-to-God addition to the static, local in $x^{-}$charge density $\rho$.

Second, note that we have not written out explicitly higher order in $a^{-}$terms in the effective action. There are of course such terms, which come from expanding the Wilson line part of the action. Disregarding these terms gives the effective action with the coupling of the field $a^{-}$to the charge density of the form $a^{-} \rho$. However, imposing gauge invariance on the final result together with the requirement that the linear in $a^{-}$ term of the gauge invariant action should coincide with the result of our calculation, the full gauge invariant form of the effective action will be recovered. In the following therefore we will concentrate on the linear term $a^{-} \rho$ only.

The procedure now is the following:

1. Integrate over $\delta A^{\mu}$ at fixed $\rho$ and fixed $\delta \rho$. 
2. Integrate over $\rho$ at fixed $\rho^{\prime}=\rho+\delta \rho$.

This generates the new effective action which formally can be written as

$$
\exp \left\{i S\left[\rho^{\prime}, a^{\mu}\right]\right\}=\exp \left\{-F^{\prime}\left[\rho^{\prime}\right]-\frac{i}{4} G^{2}(a)+i g a \rho^{\prime}\right\}
$$

with

$$
\exp \left\{-F^{\prime}\left[\rho^{\prime}\right]\right\}=\int[D \rho][D \delta A] \delta\left(\rho^{\prime}-\rho-\delta \rho[\delta A]\right) \exp \left\{-F[\rho]-\frac{i}{2} \delta A D^{-1}[\rho] \delta A\right\}
$$

Of course, to leading order in $\ln 1 / x$ only terms linear in $\alpha_{s} \ln 1 / x$ should be kept in $F^{\prime}$. Defining

$$
\alpha_{s} \ln \frac{1}{x} \Delta[\rho] \equiv F^{\prime}[\rho]-F[\rho]
$$

gives the $\mathrm{RG}$ equation

$$
\frac{d}{d y} F[\rho]=\alpha \Delta[\rho]
$$

In this way our renormalization group procedure leads to a set of evolution equations for all coefficient functions in the functional $F[\rho]$. Technically the most complicated part

of the calculation in eq.(28) turns out to be the inversion of the operator $D^{-1}$ which arises after integration over $\delta A$. The rest of the integrations in eq.(28) can be performed explicitly and it is possible to express $\Delta$ explicitly in terms of $D[\rho]$. This work is now in progress. In this paper however, we will only consider the weak field limit. In this limit $D$ can be expanded in powers of $\rho$. We will show in the next section that the evolution equations eq. (30) yield in this limit the celebrated BFKL equation.

\section{The weak field limit and the BFKL equation}

In our semiclassical approach the glue distribution function is given by (see ref. [9])

$$
g\left(x, Q^{2}\right)=\frac{1}{x} \int_{0}^{Q^{2}} d^{2} k_{t} d^{2}\left(x_{t}-y_{t}\right) e^{-i k_{t}\left(x_{t}-y_{t}\right)}<<\alpha_{a}^{i}\left(x_{t}\right) \alpha_{a}^{i}\left(y_{t}\right)>>_{\rho}
$$


where by $<<>>_{\rho}$ we denote the average over the ensemble of $\rho\left(x_{t}\right)$ with the statistical weight eq.(12). In the weak field regime the classical field $\alpha^{i}$ is related to the charge density by ([9])

$$
\alpha_{a}^{i}=-\frac{\partial^{i}}{\partial_{t}^{2}} \rho_{a}
$$

It therefore follows that

$$
x g\left(x, Q^{2}\right)=\int_{0}^{Q^{2}} \frac{d^{2} k_{t}}{k_{t}^{2}} \varphi\left(k_{t}\right)
$$

with

$$
\varphi\left(k_{t}\right)=\int d^{2}\left(x_{t}-y_{t}\right) e^{-i k_{t}\left(x_{t}-y_{t}\right)}<<\rho\left(x_{t}\right) \rho\left(y_{t}\right)>>_{\rho}
$$

The unintegrated gluon density $\varphi$ is therefore nothing but the Fourier transform of the correlation function of the color charge density. To see how the BFKL kernel arises we should therefore compute the charge density correlation function $\left\langle<\rho^{\prime} \rho^{\prime}\right\rangle_{\rho}$ after one step in our RG procedure.

One could do it of course by calculating $F^{\prime}$ first, and then averaging over $\rho$. However in the weak field case it is simpler to directly express $\left\langle<\rho^{\prime} \rho^{\prime}>>\right.$ in terms of $\langle<\rho \rho>>$ using the explicit relations eqs. (23,24,25)

$$
\begin{aligned}
<<\rho^{\prime} \rho^{\prime}>_{\rho} & =<<\left[<(\rho+\delta \rho)(\rho+\delta \rho)>_{\delta A}\right]>_{\rho} \\
& =<<\rho \rho>_{\rho}+2<<\rho<\delta \rho>_{\delta A}>_{\rho}+<<\left[<\delta \rho \delta \rho>_{\delta A}\right]>_{\rho}
\end{aligned}
$$

Here $<>_{\delta A}$ denotes averaging over the hard fluctuation field $\delta A$ at fixed value of $\rho$. The relation between the correlators becomes explicit already after averaging over $\delta A$ so that the averaging over $\rho$ does not need to be performed explicitly.

Noting that $\delta \rho_{1}$ is linear in $\delta A$ while $\delta \rho_{2}$ is quadratic in $\delta A$ (eqs.24,25) we have

$$
<\delta \rho>_{\delta A}=<\delta \rho_{2}>_{\delta A}
$$

and

$$
<\delta \rho \delta \rho>_{\delta A}=<\delta \rho_{1} \delta \rho_{1}>_{\delta A}
$$


The second equation is valid to leading order in $\alpha_{s} \ln \frac{1}{x}$. As we shall see now, eqs.(36) and (37) contribute the virtual and the real part of the BFKL kernel respectively.

\subsection{The Virtual Part}

Let us start by considering eq.(36). It has the diagrammatic representation of Fig.1.

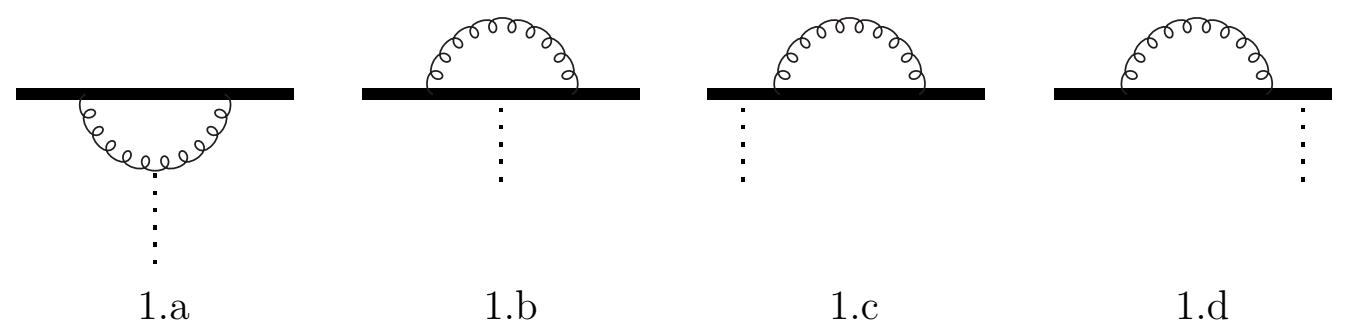

Figure 1: The virtual diagrams. The solid lines represent the effective vertex arising from expansion of the Wilson line in the action. The wavy line represents the free propagator of the hard fluctuation field $\delta A$. The dashed lines indicate the coupling of the soft fluctuation field $a^{-}$.

The diagram on Fig. 1a corresponds to the calculation of

$$
<\delta \rho_{21}^{a}(x)>_{\delta A} \equiv-g f^{a b c}<\left(\partial^{+} \delta A_{i}^{b} \delta A_{i}^{c}>_{\delta A}\right.
$$

where the propagator of the fluctuation field is expanded to first order in the color charge density $\rho$. Explicitly尸

$$
\begin{aligned}
<\left(\partial^{+} \delta A_{i}^{b}(x) \delta A_{i}^{c}(x)>_{\delta A}\right. & =\int\left[D \delta A_{i}\right]\left(\partial^{+} \delta A_{i}^{b}(x)\right) \delta A_{i}^{c}(x) \exp \left[-\frac{i}{4} G^{2}\right] \\
& \times\left[\frac{g^{2}}{N_{c}} \int d^{4} z_{t} \rho^{a}\left(z_{t}\right) \operatorname{tr} T^{a} \int^{z^{+}} d y^{+} \delta A^{-}\left(y^{+}\right) \delta A^{-}\left(z^{+}\right)\right]
\end{aligned}
$$

\footnotetext{
${ }^{2}$ There is in principle another contribution to this quantity: the standard gluon self energy diagram. It however does not give a contribution logarithmic in $x$, and for this reason is not considered here.
} 
In terms of free gluon propagators this is

$$
\begin{aligned}
<\left(\partial^{+} \delta A_{i}^{b}(x) \delta A_{i}^{c}(x)>_{\delta A}\right. & =\frac{i g^{2}}{2} f^{a b c} \int d^{4} z \rho^{a}\left(z_{t}\right) \\
& \times\left[-\partial_{x}^{+} G^{i-}\left(x^{+}-z^{+}\right) \int^{z^{+}} d y^{+} G^{i-}\left(x^{+}-y^{+}\right)\right. \\
& \left.+\int^{z^{+}} d y^{+} \partial_{x}^{+} G^{i-}\left(x^{+}-y^{+}\right) G^{i-}\left(x^{+}-z^{+}\right)\right]
\end{aligned}
$$

where only the + components of the arguments of the propagators are shown explicitly since the transverse coordinates are all equal, and $y^{-}=z^{-}=0$. Here

$$
\begin{aligned}
G^{--}(p) & =\frac{2 i p^{-}}{p^{+} p^{2}} \\
G^{-i}(p) & =\frac{i p_{i}}{p^{+} p^{2}}
\end{aligned}
$$

Performing the $z^{+}, z^{-}, y^{+}$and $y^{-}$integrations we obtain

$$
\begin{array}{r}
<\left(\partial^{+} \delta A_{i}^{b}(x) \delta A_{i}^{c}(x)>_{\delta A}=-i g^{2} f^{a b c} \int d^{2} z_{t} \rho^{a}\left(z_{t}\right) \int \frac{d^{2} q_{t}}{(2 \pi)^{2}} \frac{d^{4} p}{(2 \pi)^{4}} e^{i\left(p_{t}+q_{t}\right)\left(x_{t}-z_{t}\right)}\right. \\
\times\left[\frac{p_{t} \cdot q_{t}}{p^{+}\left(p^{-}-i \epsilon\right)\left(p^{2}+i \epsilon\right)\left(2 p^{-} p^{+}-q_{t}^{2}+i \epsilon\right)}\right]
\end{array}
$$

The $i \epsilon$ prescription for the $p^{-}$pole follows from adding a convergence factor to the exponentials when performing integrals of the form

$$
\int_{-\infty}^{z^{+}} d y^{+} e^{i p^{-} y^{+}}
$$

so that there is no contribution from infinity. Also the $p^{+}$integral is restricted to run between the lower and upper limits of the momentum region where the hard fluctuation fields are being integrated out.

Closing the integration contour in the complex $p^{-}$plane, the $p^{-}$integration is easily performed. The $p^{+}$integral then factors out as $\int_{P_{n}^{+}}^{P_{n}^{+}} \frac{d p^{+}}{p^{+}}$which gives $\ln \frac{P_{n-1}^{+}}{P_{n}^{+}} \equiv \ln \frac{x_{n-1}}{x_{n}}$.

Finally

$$
<\left(\partial^{+} \delta A_{i}^{b}(x) \delta A_{i}^{c}(x)>_{\delta A}=\frac{g^{2}}{2 \pi} f^{a b c} \ln \frac{x_{n-1}}{x_{n}} \int d^{2} z_{t} \rho^{a}\left(z_{t}\right) \int \frac{d^{2} q_{t}}{(2 \pi)^{2}} \frac{d^{2} p_{t}}{(2 \pi)^{2}} \frac{p_{t} \cdot q_{t}}{p_{t}^{2} q_{t}^{2}} e^{i\left(p_{t}+q_{t}\right)\left(x_{t}-z_{t}\right)}\right.
$$


Fourier transforming the above equation and shifting $p_{t} \rightarrow p_{t}-q_{t}$ gives

$$
<\delta \rho_{21}^{a}\left(k_{t}\right)>_{\delta A}=-\frac{g^{2} N_{c}}{(2 \pi)^{3}} \ln \frac{x_{n-1}}{x_{n}} \rho^{a}\left(k_{t}\right)\left[\frac{k_{t}^{2}}{2} \int \frac{d^{2} p_{t}}{p_{t}^{2}\left(p_{t}-k_{t}\right)^{2}}-\int \frac{d^{2} p_{t}}{p_{t}^{2}}\right]
$$

The contribution of Figs. 1(b,c,d) to the color charge density is

$$
\begin{aligned}
<\delta \rho_{22}^{a}\left(x_{t}\right)>_{\delta A} & =-g^{2} N_{c} \rho^{a}\left(x_{t}\right) \int d y^{+} d z^{+} G^{--}\left(y^{+}-z^{+}\right)\left[\theta\left(z^{+}-y^{+}\right) \theta\left(y^{+}-x^{+}\right)\right. \\
& \left.+\theta\left(x^{+}-z^{+}\right) \theta\left(z^{+}-y^{+}\right)+\frac{1}{2} \theta\left(z^{+}-x^{+}\right) \theta\left(x^{+}-y^{+}\right)\right]
\end{aligned}
$$

This is the average of the rest of the terms in eq.(25) with all gloun propagators taken to be free propagators. The different theta functions represent the different order of emision of the soft field $a^{-}\left(x^{+}\right)$. The first two terms correspond to diagrams where the soft field is emitted either before or after the hard fluctuation has been emitted and reabsorbed, while the last term corresponds to emission during the time the hard fluctuation was "in flight". The factor $1 / 2$ in the third term is the result of color algebra. Using the identity

$$
\theta\left(z^{+}-y^{+}\right) \theta\left(y^{+}-x^{+}\right)+\theta\left(x^{+}-z^{+}\right) \theta\left(z^{+}-y^{+}\right)+\theta\left(z^{+}-x^{+}\right) \theta\left(x^{+}-y^{+}\right)=\theta\left(z^{+}-y^{+}\right)
$$

this expression can be written as

$$
\begin{aligned}
<\delta \rho_{22}^{a}\left(x_{t}\right)>_{\delta A}=-g^{2} & N_{c} \rho^{a}\left(x_{t}\right) \int d y^{+} d z^{+} G^{--}\left(y^{+}-z^{+}\right) \\
& \times\left[\theta\left(z^{+}-y^{+}\right)-\frac{\theta\left(z^{+}-x^{+}\right) \theta\left(x^{+}-y^{+}\right)}{2}\right]
\end{aligned}
$$

The first term which is totally independent of $x^{+}$, vanishes since it is proportional to $G^{--}\left(p^{-}=0\right)$ and $G^{--}\left(p^{-}\right) \sim \frac{p^{-}}{p^{+} p^{2}}$ We therefore obtain

$$
<\delta \rho_{22}^{a}\left(x_{t}\right)>_{\delta A}=\frac{g^{2} N_{c}}{2} \rho^{a}\left(x_{t}\right) \int d y^{+} d z^{+} G^{--}\left(y^{+}-z^{+}\right) \theta\left(z^{+}-x^{+}\right) \theta\left(x^{+}-y^{+}\right)
$$

After Fourier transforming to momentum space and using the explicit form of the free propagator for $G^{--}$this yields

$$
<\delta \rho_{22}^{a}\left(k_{t}\right)>_{\delta A}=-\frac{g^{2} N_{c}}{(2 \pi)^{3}} \ln \frac{x_{n-1}}{x_{n}} \rho^{a}\left(k_{t}\right) \int \frac{d^{2} p_{t}}{p_{t}^{2}}
$$


Combining equations (44) and (49) gives

$$
<\delta \rho_{2}^{a}\left(k_{t}\right)>_{\delta A}=-\frac{g^{2} N_{c}}{2(2 \pi)^{3}} \ln \frac{x_{n-1}}{x_{n}} \rho^{a}\left(k_{t}\right) \int d^{2} p_{t} \frac{k_{t}^{2}}{p_{t}^{2}\left(p_{t}-k_{t}\right)^{2}}
$$

This is the contribution of all virtual diagrams to the change in the color charge density.

\subsection{The Real Part}

Now we have to compute the connected correlator of $\delta \rho_{1}$

$$
\delta \rho^{a}=-2 f^{a b c} \alpha_{i}^{b} \delta A_{i}^{c}-\frac{g}{2} f^{a b c} \rho^{b}\left(x_{t}\right) \int d y^{+}\left[\theta\left(y^{+}-x^{+}\right)-\theta\left(x^{+}-y^{+}\right)\right] \delta A^{-c}\left(y^{+}\right)
$$

The two theta functions in the second term again correspond to emmision of the soft field before and after the emmision of the hard fluctuation respectively.

The procedure to compute $<\delta \rho_{1}^{a}\left(x_{t}\right) \delta \rho_{1}^{a}\left(y_{t}\right)>_{\delta A}$ is as before; we contract the hard fluctuation fields $\delta A$ and use free propagators for $\left\langle\delta A \delta A>_{\delta A}\right.$. Squaring the first term in eq. (51) gives

$$
<\delta \rho_{11}^{a}\left(x_{t}\right) \delta \rho_{11}^{a}\left(y_{t}\right)>_{\delta A}=\frac{2 N_{c}}{(2 \pi)^{3}}(2 \pi)^{2} \delta^{2}\left(x_{t}-y_{t}\right) \ln \frac{x_{n-1}}{x_{n}} \alpha_{i}^{a}\left(x_{t}\right) \alpha_{i}^{a}\left(y_{t}\right)
$$

This contribution diagrammatically is depicted on Fig. 2.a.

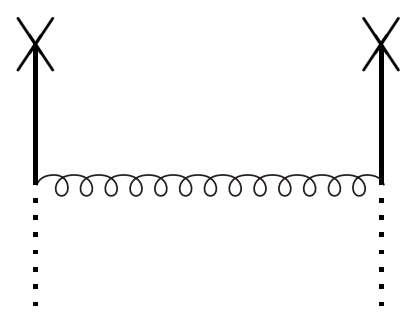

Figure 2.a: The diagram for the contribution of eq. (59). The crosses denote insertions of the background field $\alpha_{i}$. The rest of the notations are as in Fig.1.

Squaring the second term (Fig. 2.b) gives

$$
<\delta \rho_{12}^{a}\left(x_{t}\right) \delta \rho_{12}^{a}\left(y_{t}\right)>_{\delta A}=\frac{2 g^{2} N_{c}}{(2 \pi)^{3}} \ln \frac{x_{n-1}}{x_{n}} \rho^{a}\left(x_{t}\right) \rho^{a}\left(y_{t}\right) \int \frac{d^{2} p_{t}}{p_{t}^{2}} e^{i p_{t}\left(x_{t}-y_{t}\right)}
$$



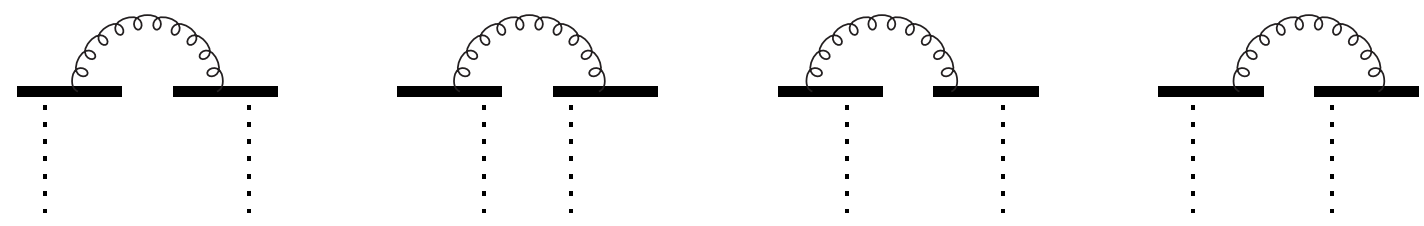

Figure 2.b: Contribution of eq. (53).

while the contribution of the cross term (Fig. 2.c) is

$$
2<\delta \rho_{12}^{a}\left(x_{t}\right) \delta \rho_{21}^{a}\left(y_{t}\right)>_{\delta A}=\frac{4 i g N_{c}}{(2 \pi)^{3}} \ln \frac{x_{n-1}}{x_{n}} \alpha_{i}^{a}\left(x_{t}\right) \rho^{a}\left(y_{t}\right) \int \frac{d^{2} p_{t}}{p_{t}^{2}} p_{i} e^{i p_{t}\left(x_{t}-y_{t}\right)}
$$
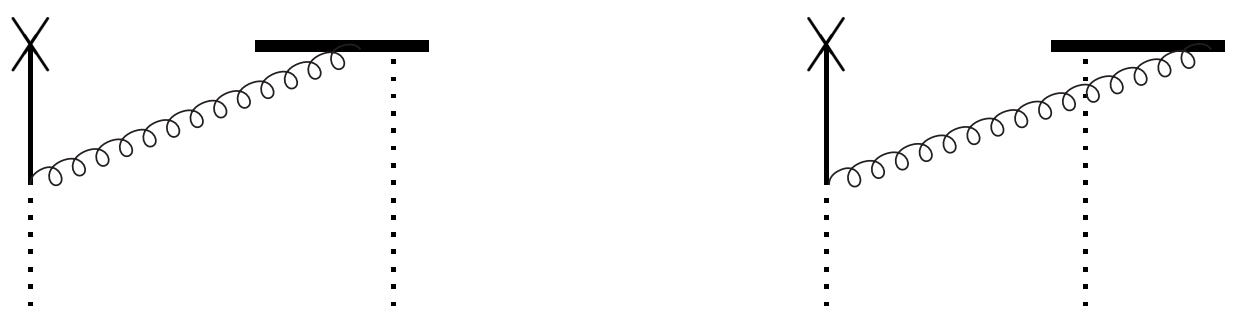

Figure 2.c: Contribution of eq. (54).

Fourier transforming to momentum space, using

$$
\alpha_{i}^{a}\left(k_{t}\right)=-i g \frac{k_{i}}{k_{t}^{2}} \rho^{a}\left(k_{t}\right)
$$

and combining the three contributions we obtain

$$
\begin{aligned}
<\delta \rho_{1}^{a}\left(k_{t}\right) \delta \rho_{1}^{a}\left(-k_{t}\right)>_{\delta A}= & \frac{2 g^{2} N_{c}}{(2 \pi)^{3}} \ln \frac{x_{n-1}}{x_{n}} \int d^{2} p_{t} \rho^{a}\left(p_{t}\right) \rho^{a}\left(-p_{t}\right) \\
& {\left[\frac{1}{p_{t}^{2}}+\frac{1}{\left(p_{t}-k_{t}\right)^{2}}-2 \frac{p_{t} \cdot\left(p_{t}-k_{t}\right)}{p_{t}^{2}\left(p_{t}-k_{t}\right)^{2}}\right] }
\end{aligned}
$$


The use of the identity

$$
-2 \frac{p_{t} \cdot\left(p_{t}-k_{t}\right)}{p_{t}^{2}\left(p_{t}-k_{t}\right)^{2}}=-\frac{1}{p_{t}^{2}}-\frac{1}{\left(p_{t}-k_{t}\right)^{2}}+\frac{k_{t}^{2}}{p_{t}^{2}\left(p_{t}-k_{t}\right)^{2}}
$$

gives

$$
<\delta \rho_{1}^{a}\left(k_{t}\right) \delta \rho_{1}^{a}\left(-k_{t}\right)>_{\delta A}=\frac{2 g^{2} N_{c}}{(2 \pi)^{3}} \ln \frac{x_{n-1}}{x_{n}} \int d^{2} p_{t} \rho^{a}\left(p_{t}\right) \rho^{a}\left(-p_{t}\right) \frac{k_{t}^{2}}{p_{t}^{2}\left(p_{t}-k_{t}\right)^{2}}
$$

This is the contribution of all real diagrams to the change in charge density.

Inserting the expressions eqs.(58) and (50) into equation (36) we obtain

$$
\begin{aligned}
<<\rho^{\prime}\left(k_{t}\right) \rho^{\prime}\left(-k_{t}\right)>>_{\rho}-<<\rho\left(k_{t}\right) \rho\left(-k_{t}\right)>>_{\rho} \\
=-\frac{g^{2} N_{c}}{(2 \pi)^{3}} \ln \frac{x_{n-1}}{x_{n}} \int d^{2} p_{t} \frac{k_{t}^{2}}{p_{t}^{2}\left(p_{t}-k_{t}\right)^{2}} \\
\quad \times\left[<<\rho^{a}\left(k_{t}\right) \rho^{a}\left(-k_{t}\right)>_{\rho}-2<<\rho^{a}\left(p_{t}\right) \rho^{a}\left(-p_{t}\right)>>_{\rho}\right]
\end{aligned}
$$

Identifying $<<\rho\left(k_{t}\right) \rho\left(-k_{t}\right)>>_{\rho}=\varphi\left(y, k_{t}\right)$, and $<<\rho^{\prime}\left(k_{t}\right) \rho^{\prime}\left(-k_{t}\right)>_{\rho}=\varphi\left(y+d y, k_{t}\right)$ as in eq. (34), the equation (59) can be rewritten in differential form to give precisely the BFKL equation eq.(2).

\section{Discussion}

We have shown in this paper that the RG equation of our low $x$ Wilson renormalization group procedure reduces to the BFKL equation in the weak field limit. This in itself is very satisfying and encourages us to continue the study of our low $x$ effective action.

It also demonstrates the relation of the semiclassical aproach of [9], [11] in its present form to other more conventional approaches to low $x$ physics. In this connection it is specially illuminating to compare it with the effective action approach of Lipatov 12 . In fact, an attentive reader must have noticed a lot of similarities between our effective action eq. (77) and the one used by Lipatov for description of the high energy scattering in the multi-Regge limit of QCD [4], [12] (cf. Eqs. 210, 249 in [四]). 


$$
\begin{aligned}
S_{\text {Lipatov }} & =\partial_{\mu} R_{a}^{-} \partial^{\mu} R_{a}^{+}-\frac{1}{4} G^{2} \\
& +\frac{i}{N_{c}} \partial^{2} R_{a}^{+}(x) \operatorname{tr} T_{a} W_{x^{+}=-\infty, x^{+}=\infty}\left[A^{-}\right] \\
& +\frac{i}{N_{c}} \partial^{2} R_{a}^{-}(x) \operatorname{tr} T_{a} W_{x^{-}=-\infty, x^{-}=\infty}\left[A^{+}\right]
\end{aligned}
$$

where

$$
\partial^{+} R^{-}=\partial^{-} R^{+}=0
$$

This action describes gluons $A^{\mu}$ interacting with reggeons $R^{+}$and $R^{-}$corresponding to the quasistatic gluon fields dominating the $t$ - channel exchange in the high energy scattering in multiregge kinematics. Our static color charge density obviously plays the role of the reggeon fields. More explicitly, by comparing the interaction terms in (7) and (61) one should identify $\rho\left(x_{t}\right) \delta\left(x^{-}\right)$with $\partial^{2} R^{+}$. The Wilson line term, which describes the interaction of the reggeon with gluons is otherwise the same in eqs.(17) and (61). The calculational strategy is actually similar up to a point. In both approaches one first solves for a gluon field in a given reggeon (classical charge) background and then expands the action around this classical configuration.

There are however some conceptual as well as technical differences between the two approaches. As far as we can tell the reason for this is twofold. First our action is constructed to describe the structure of one source of color field (nucleus or hadron DIS structure function) and not the collision of two hadrons. In our case only one component of the reggeon field (charge density) does not vanish, and $R^{-}=0$. Secondly although one is tempted at first to identify the $\partial_{\mu} R_{a}^{+} \partial^{\mu} R_{a}^{-}$(reggeon propagator) term in eq.(61) with our $F[\rho]$, the two terms in fact represent very different physics. As discussed in Section $2, F[\rho]$ is the statistical weight with which a given charge density (reggeon) configuration is present in the hadronic wave function. Consequently, it appears as an imaginary part 
of the action. The reggeon propagator term in $S_{\text {Lipatov }}$ on the other hand is real and is included to reproduce the Born term in the scattering amplitude of one hadron in the

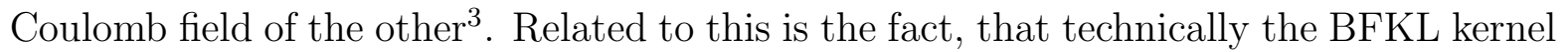
appears quite differently in the two calculations. In our case the action calculated on the solution of classical equations of motion vanishes. The contribution to the kernel therefore entirely comes from the small fluctuation integral. In the case of $S_{\text {Lipatov }}$, 12] it is only the virtual part of BFKL kernel that comes from the integral over the small fluctuation. The real part of the kernel according to [12 appears already in the action calculated on the classical solution.

We would also like to note, that due to the fact that in our approach $J^{-}$vanishes, the classical solution is simpler. We are hoping therefore that the small fluctuation propagator in this classical background can be calculated explicitly along the lines of [10].

The low $x$ renormalization group procedure described in the present paper and in [11] is a new element and it would be also interesting to incorporate it in the analysis of $S_{\text {Lipatov }}$. Here we want to make one remark about the nature of this renormalization group procedure. It looks different from the standard RG used in the analysis of DIS at moderate $x$, in that it is the change in the longitudinal rather than the transverse momentum scale that defines the block spin procedure. Conceptually however, the two are very similar. It is more physical to think about the modes which are being integrated out not in terms of spatial momentum, but rather in terms of the frequency $p^{-}$. The leading contribution to the scattering cross section comes from the interaction of the external probe with the quark and gluon modes which are practically static during the interaction time. The influence of the modes with frequencies higher than the inverse interaction time of the scattering process averages to zero. When the interaction time

\footnotetext{
${ }^{3}$ In fact it seems to us that besides the reggeon kinetic term the weight factor for different reggeon configurations should be present also in the two hadron case.
} 
becomes smaller, more and more field modes behave like static fields from the viewpoint of the external probe. The intensity of the "static" field therefore effectively grows as the time of the interaction decreases.

Both lowering $x$ at fixed $Q^{2}$ and raising $Q^{2}$ at fixed $x$ in DIS result in shorter interaction time between the hadron and the external probe. This is precisely the physical mechanism which underlies the growth of the partonic distributions both at smal $x$ and at large $Q^{2}$. Roughly one can think of the on shell frequency $p^{-}=\frac{p_{t}^{2}}{p^{+}}$as defining the relevant time scale for quantum fluctauations with given spatial momentum. When the longitudinal momenta of all the relevant modes are of the same order the frequency is determined entirely by the transverse momentum. The evolution in frequency then becomes the evolution in $Q^{2}$. This is the situation in the DIS at moderate $x$. If all the transverse momenta are of the same order, the frequency is governed by the longitudinal momentum. This is the case of the low $x$ renormalization group discussed in this paper.

The interchangeability of $p^{-}$and $1 / p^{+}$breaks down however, if the interaction spreads over wide range of transverse momenta. This seems to be the case in the BFKL evolution, the asymptotic solution for which has the character of a random walk in the transverse momentum space [13]. It would be interesting to formulate the unifying RG procedure, which uses the frequency directly as the RG evolution parameter. Potentially this could cure the problem with the low $k_{t}$ modes in the BFKL evolution, since in this kind of procedure those, being low frequency modes should apear in the initial condition rather than in the evolution itself.

To summarize, in this paper we have considered the renormalization group approach to the low $x$ effective action in the weak field regime. Clearly the more interesting and more complicated part of the problem is to penetrate the nonlinear regime, where the fluctuation field propagator has to be computed to all orders in $\rho$. This work is now in progress. 
Acknowledgements We are very grateful to Larry McLerran for numerous discussions on a variety of topics related to the subject of this paper and for his constant encouragement and interest in this work. We have also benefitted from discussions with M. Gyulassy, Yu. Kovchegov, A. Makhlin, B. Mueller, J.-W. Qiu, R. Rodriguez, E.

Surdutovich, R. Venugopalan and G. Zinovjev. We thank INT, University of Washington for hospitality and financial support during the program "Ultrarelativistic Nuclei: from structure functions to the quark-gluon plasma" where part of this work was done. The work of A.L. was partially supported by Russian Fund for Basic Research, Grant 96-02-16210.

\section{References}

[1] E.M. Levin, "The Pomeron: Yesterday, Today and Tomorrow", CBPF-NF010/95, hep-ph/9503399.

[2] H1 Collaboration, I. Abe et al., Nucl. Phys. B407 (1993), 515; T. Ahmed et al., Nucl. Phys. B439 (1995), 471; ZEUS Collaboration, M. Derrick et al., Phys. Lett. B316 (1993), 412; Z. Phys. C65 (1995), 379.

[3] E.A. Kuraev, L.N. Lipatov and V.S. Fadin, Sov. Phys. JETP 45 (1977), 199; Ya.Ya. Balitsky and L.N. Lipatov, Sov. J. Nucl. Phys. 28 (1978), 22.

[4] L.N. Lipatov, "Small-x Physics in Perturbative QCD", hep-ph/9610276.

[5] V. Del Duca, "Introduction to the Perturbative Pomeron and to Jet Production at Large Rapidities", hep-ph/9503226.

[6] L.V. Gribov, E.M. Levin and M.G. Ryskin, Phys. Rep 100 (1981).

[7] J. Bartels, Nucl. Phys. B175 (1980) 365; J. Kwiecinski and M. Praszalowicz Phys. Lett. 94B (1980) 413; G.P. Korchemsky, Nucl. Phys. B462, (1996) 333. 
[8] A. Mueller, Nucl. Phys. B437 (1995), 107.

[9] L. McLerran and R. Venugopalan, Phys. Rev. D49 (1994), 2233; D49 (1994), 3352; D49 (1995), 2225.

[10] A. Ayala, J. Jalilian-Marian, L. McLerran and R. Venugopalan, Phys. Rev. D52 (1995), 2935; D53 (1996), 458.

[11] J. Jalilian-Marian, A. Kovner, L. McLerran and H. Weigert, "The Intrinsic Glue Distribution at Very Small x", hep-ph/9606337; J. Jalilian-Marian, "Saturation of Gluon Density at Small x ", hep-ph/9609350.

[12] L.N. Lipatov, Nucl. Phys. B452 (1995), 369; V.S. Fadin and L.N. Lipatov, Nucl.Phys. B477, (1996), 767.

[13] A.J. Askew, J. Kwiecinski, A.D. Martin and P.J. Sutton Phys.Rev. D49 (1994), 4402 ; 